\title{
Low-temperature carrier transport across InGaN multiple quantum wells: Evidence of ballistic hole transport
}

\author{
Saulius Marcinkevičius $\odot,{ }^{1}$ Rinat Yapparov $\odot,{ }^{1}$ Leah Y. Kuritzky, ${ }^{2}$ Yuh-Renn Wu, ${ }^{3}$ Shuji Nakamura, ${ }^{2}$ and James S. Speck ${ }^{2}$ \\ ${ }^{1}$ Department of Applied Physics, KTH Royal Institute of Technology, Electrum 229, 16440 Kista, Sweden \\ ${ }^{2}$ Materials Department, University of California, Santa Barbara, California 93106, USA \\ ${ }^{3}$ Graduate Institute of Photonics and Optoelectronics and Department of Electrical Engineering, \\ National Taiwan University, Taipei 10617, Taiwan
}

(Received 8 August 2019; revised manuscript received 5 November 2019; accepted 22 January 2020; published 18 February 2020)

\begin{abstract}
Carrier transport across polar $n$-type $\mathrm{InGaN} / \mathrm{GaN}$ multiple quantum wells (MQWs) has been studied by time-resolved photoluminescence (PL) using an optical marker technique. Efficiency of the hole transfer into the marker well experienced a nonmonotonous temperature dependence. First, as the temperature was lowered below room temperature, the number of transferred holes decreased because of the decreased efficiency of the thermionic emission. However, when the temperature was lowered below $\sim 80 \mathrm{~K}$, the number of transferred holes experienced a significant rise. In addition, the low-temperature hole transport across the MQW structure was very fast, $<3$ ps. These features indicate that the low-temperature hole transport across the MQWs is ballistic or quasiballistic. Comparison of PL data for structures with different MQW parameters suggests that at low temperatures the hole mean-free path is about $10 \mathrm{~nm}$. Probably, hole transport via light hole and split-off valence bands contributes to this high value.
\end{abstract}

DOI: 10.1103/PhysRevB.101.075305

Interwell carrier transport and carrier distribution between quantum wells (QWs) that form active regions of lightemitting diodes (LEDs) have a large impact on high-power LED performance. In case of a slow (compared to recombination) hole transport, carrier distribution between the QWs is nonuniform, which results in their accumulation in QWs closest to the $p$ side of the structure and enhanced detrimental Auger recombination. This issue is especially critical for material systems with deep wells such as $\operatorname{In}_{x} \mathrm{Ga}_{1-x} \mathrm{~N} / \mathrm{GaN}$ QWs with $x \gtrsim 0.1[1,2]$.

The carrier transport across multiple QW (MQW) structures may proceed with or without capture into the wells. In the first case, injected carriers are captured into the first QW, and their transport to the subsequent wells proceeds either by thermionic transport (a succession of capture into and thermionic emission out of the QWs) or tunneling, or a combination of those [3]. The transport mechanism without the carrier capture is ballistic or quasiballistic (with a little change in the propagation direction upon scattering with a phonon) $[3,4]$.

The prevalence of one or another transport mechanism depends on the carrier type, structure parameters, and external conditions. For electrons at room temperature all transport mechanisms, including the ballistic transport, are relevant [4].

Published by the American Physical Society under the terms of the Creative Commons Attribution 4.0 International license. Further distribution of this work must maintain attribution to the author(s) and the published article's title, journal citation, and DOI. Funded by Bibsam.
For holes, only the thermionic emission has to be considered $[2,5]$. As the temperature is decreased, the thermionic emission rate decreases exponentially and the interwell transport stops at $\sim 200 \mathrm{~K}$ [2]. Surprisingly, as the temperature is lowered further, the hole transfer over MQWs starts increasing and at $4 \mathrm{~K}$ becomes very efficient. This suggests that at low temperatures a transport mechanism other than the thermionic emission turns on. In this paper, we analyze this unexpected effect.

The interwell carrier transport was measured by timeresolved photoluminescence (PL) in InGaN/GaN MQW structures containing a deeper marker well (inset to Fig. 2). The structures were grown by metal-organic chemical vapor deposition on $c$-plane sapphire substrates. Counting from the substrate side, the structures consist of a $6-\mu \mathrm{m}$ buffer layer, a deep 3-nm $\mathrm{In}_{0.18} \mathrm{Ga}_{0.82} \mathrm{~N} \mathrm{QW}$, five shallow 3-nm $\mathrm{In}_{0.12} \mathrm{Ga}_{0.88} \mathrm{~N}$ QWs, and a $100-\mathrm{nm}$ GaN cap layer. The barrier widths are $5.5,8.5$, and $12.5 \mathrm{~nm}$ for thin, medium, and thick barrier structures. A structure with three shallow QWs and 8.5-nm barriers was also examined. PL peak wavelengths for the shallow and deep QWs are in the region of 420-430 and 460-480 nm, respectively. The samples were not intentionally doped with the majority electron concentration of about $1 \times 10^{17} \mathrm{~cm}^{-3}$.

In most experiments, carriers were excited by 300 -fs pulses with 260-nm central wavelength (third harmonic of a selfmode-locking Ti:sapphire laser). Because of the small absorption length in $\mathrm{GaN}(\sim 50 \mathrm{~nm})$, most of the carriers were excited in the cap layer. Carrier distribution between the QWs was evaluated by measuring time-integrated PL spectra and PL transients at shallow and deep QW emission wavelengths. To measure fast carrier dynamics, a spectrometer-streak camera system with a temporal resolution of 3 ps was used. 


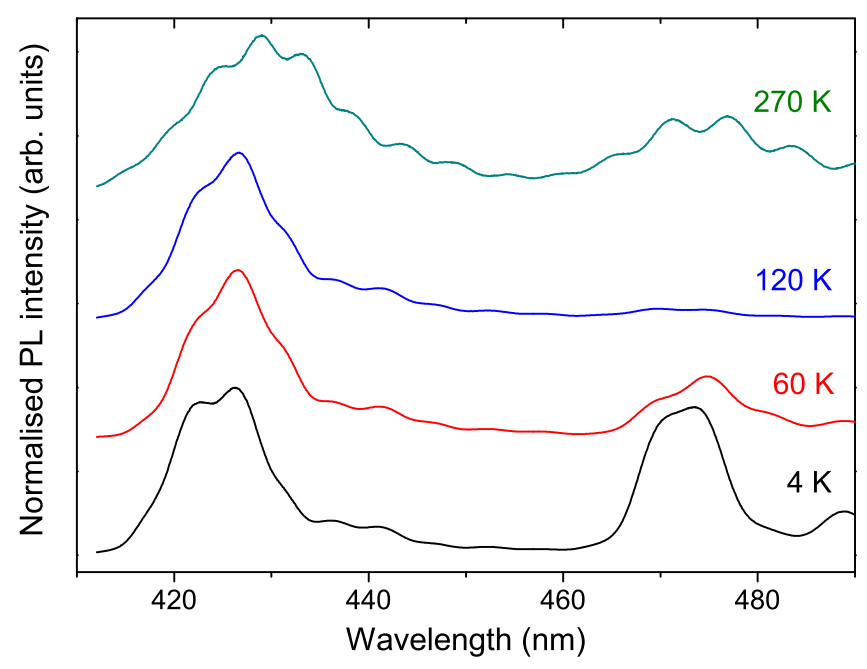

FIG. 1. PL spectra of the thin barrier structure at different temperatures. Oscillatory pattern of the spectra is due to Fabry-Perot interference.

To record PL transients on a long (tens of ns) timescale, the laser pulse repetition rate was reduced from 80 to $4 \mathrm{MHz}$, and PL dynamics were measured by time-correlated single-photon counting (temporal response $50 \mathrm{ps}$ ). In the latter case, emissions from shallow and deep QWs were separated by bandpass filters. Time-integrated PL spectra were recorded along with the transients using a spectrometer with a liquid nitrogen cooled charge-coupled device detector array. Measurements were performed in the 4-300 $\mathrm{K}$ temperature range with a focus on low temperatures. Photoexcited pulse energy varied between 2.5 and $25 \mathrm{pJ}$, which corresponds to an average photoexcited carrier density from $5 \times 10^{16}$ to $5 \times 10^{17} \mathrm{~cm}^{-3}$.

Figure 1 shows PL spectra of the thin $(5.5-\mathrm{nm})$ barrier structure. At $270 \mathrm{~K}$, the deep QW PL peak is predominantly generated by carriers arriving from the cap layer [2]. At $120 \mathrm{~K}$, the thermionic transport is suppressed, and this peak reflects recombination of carriers that have been excited directly in the deep QW and adjacent barriers (about $4 \%$ of all photoexcited carriers). As the temperature is lowered below $\sim 80 \mathrm{~K}$, the deep QW peak increases until at $4 \mathrm{~K}$ the shallow and deep QW PL peaks become of similar intensities. This suggests that at low temperatures carrier transfer to the deep QW is increased.

For more quantitative data on the carrier distribution between the shallow and deep QWs, one should take into account that spectrally integrated PL peak intensities $I_{\mathrm{PL}}$ are proportional not only to the number of carriers $n$ in the respective wells but also to internal quantum efficiencies (IQE) $\eta\left(I_{\mathrm{PL}} \propto n \eta\right)$, which may be different for shallow and deep QWs. To evaluate the ratio of the number of carriers in the deep $n_{d}$ and shallow $n_{\text {sh }}$ QWs, IQE values were estimated from temperature-dependent PL decay times and transient amplitudes measured using direct carrier excitation into the QWs at $390 \mathrm{~nm}$. In the estimation procedure it was considered that at short times after the excitation PL decay at $4 \mathrm{~K}$ is determined solely by the radiative recombination, and that the inverse PL transient amplitude is proportional to the radiative recombination time [6,7].

Figure 2 shows the temperature dependence of $n_{d} / n_{\mathrm{sh}}$. The PL intensity and IQE of the shallow QWs at temperatures

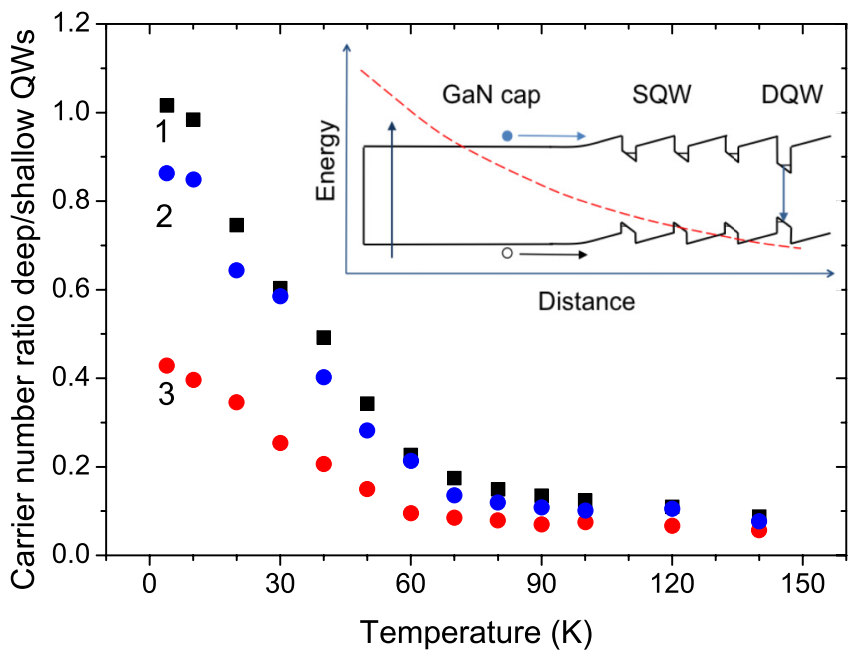

FIG. 2. Ratio of carrier numbers in the deep and shallow QWs $n_{d} / n_{\mathrm{sh}}$ for structures with thin (1), medium (2), and thick (3) barriers. The inset shows schematics of the experiment with the red dashed line illustrating the carrier excitation profile. The layer thicknesses are not in scale. SQW and DQW stand for shallow and deep QWs, respectively.

below $100 \mathrm{~K}$ were found to be temperature independent with a PL decay time of about $4 \mathrm{~ns}$. Hence, the increase of $n_{d} / n_{\mathrm{sh}}$ with decreasing temperature indicates that the number of carriers transferred to the deep QW becomes larger. The increase of $n_{d} / n_{\mathrm{sh}}$ is largest for the narrow barrier structure. In the medium barrier structures with 5 and 3 (not shown) shallow QWs, $n_{d} / n_{\mathrm{sh}}$ is slightly lower, while for the thick barriers it is about twice smaller. In all cases, increase of $n_{d} / n_{\mathrm{sh}}$ with decreased temperature is nearly linear. With increased pulse energy (from 2.5 to $25 \mathrm{pJ}$ ),$n_{d} / n_{\mathrm{sh}}$ experiences a slight decrease of $10-20 \%$.

Deep QW PL dynamics at several temperatures is shown in Fig. 3. At high temperature, the PL increase is composed of a fast and slow component. The fast one with a rise time $<3$ ps (limited by the experimental resolution of the streak

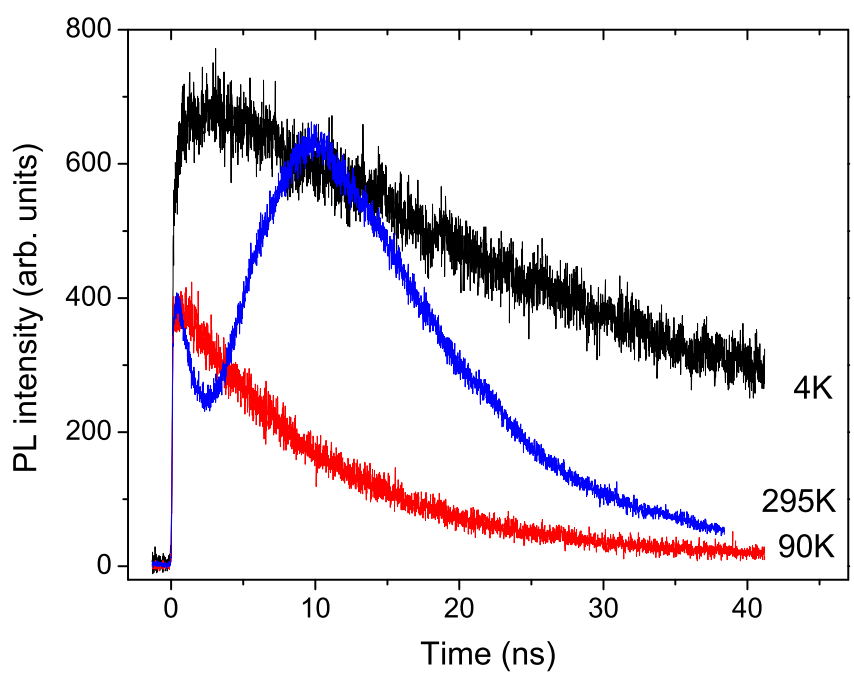

FIG. 3. PL transients of the thin barrier sample at 4, 90, and $295 \mathrm{~K}$. 
camera) reflects direct carrier capture into the deep QW from the adjacent barriers and relaxation within this QW. The slow component with a rise time of $4.5 \mathrm{~ns}$ originates from carriers arriving at the deep QW via thermionic transport [2]. At intermediate temperatures $(90 \mathrm{~K})$, the thermionic transport is suppressed and only the fast component, determined by the directly excited carriers, remains. At $4 \mathrm{~K}$, the PL rise time is still short $(<3 \mathrm{ps})$ but its amplitude $I_{\max }$ is considerably enhanced. For the resonant excitation directly into the deep QW no such enhancement is observed. On the contrary, at $4 \mathrm{~K} I_{\max }$ is slightly lower than at temperatures in the $20-80 \mathrm{~K}$ range, which may be attributed to decrease of the radiative lifetime $\tau_{r}$ due to the carrier delocalization and reduced electron and hole spatial separation in the QW plane at elevated temperatures $\left(I_{\max } \sim 1 / \tau_{r}\right)[8]$.

The increased $n_{d} / n_{\mathrm{sh}}$ and the short PL rise time at low temperatures point at an efficient and rapid carrier transfer from the GaN cap to the deep QW. Self-consistent simulation of Schrödinger-Poisson equations shows that, in spite of the unintentional $n$-type doping, before excitation the deep QW is depleted of carriers. Thus, to observe the deep QW PL with a short rise time, both electrons and holes should rapidly transfer to this well. For electrons, such transport over QWs, often referred to as an electron overflow, is common [4]. Hence, the critical phenomenon leading to the fast rise and large intensity of the deep QW PL is the transport of holes. Here one should bear in mind that the experimentally determined transfer time (PL rise time) is significantly shorter than the exciton formation time of $\sim 10$ ps $[9,10]$, which means that electrons and holes travel toward the deep QW as free carriers.

To reveal the mechanism of the low-temperature hole transfer to the deep QW, let us discuss several possibilities. First, let us consider transport mechanisms with capture into shallow QWs. The most efficient transport mechanism at high temperatures, the thermionic transport during which the transport takes place via subsequent capture and thermionic emission events, is suppressed [2]. Second, hole tunneling through shallow QWs, especially for barriers as wide as $12.5 \mathrm{~nm}$, is hardly probable and would certainly take much longer that a few ps. A time-resolved PL study of asymmetric $a$-plane InGaN QWs with 5.5 to 7.2-nm barriers has shown that in these QWs tunneling occurs on a 100-ps timescale [11]. Obviously, it should take even longer for 8.5 and 12.5 -nm barriers of our structures. The third mechanism to consider is Auger-assisted interwell transfer. This effect has been demonstrated by steady-state PL measurements in structures with QWs emitting at different wavelengths [12]. In that work, the timescale of the process was not explored; however, Auger-assisted interwell transport should proceed at the rate of Auger recombination. Previously, the Auger-dominated recombination time at $4 \mathrm{~K}$ was found to be about $100 \mathrm{ps}$ for carrier densities of $\sim 5 \times 10^{19} \mathrm{~cm}^{-3}$ [13]. In our experiments, recombination times are long, several ns for shallow and up to $50 \mathrm{~ns}$ for deep QWs, and the carrier densities are much lower. In addition, the Auger-assisted transfer rate would have a strong dependence on the carrier density, which is not observed in our experiments.

One should also note that reabsorption of light in the deep QW emitted during recombination of carriers captured to the shallow QWs (photon recycling) cannot account for the fast and efficient carrier transport to the deep QW because of the timescale and efficiency of the photon recycling. The timescale of the photon recycling is determined by the recombination time in the shallow QWs, which is in the ns range. Moreover, because of the small deep QW width $(3 \mathrm{~nm}$ compared to the absorption length of $100 \mathrm{~nm}$ ), no more than $\sim 3 \%$ of radiation emitted from the shallow QWs could be absorbed by the deep QW, which contradicts our data.

Thus, neither of the effects that involve carrier capture into shallow QWs can account for the rapid interwell hole transport. Elimination of these possibilities leaves ballistic transport over shallow QWs without capture as the only viable alternative that could explain our experimental results. As mentioned above, the critical process is the hole transfer. In principle, the fast hole transfer might be aided by the photo-Dember effect, which implies that photoexcited electrons, because of their smaller mass and larger mobility, move faster toward the depth of the structure and, due to Coulomb interaction, drag holes. However, the photo-Dember effect is most efficient at high carrier excess energies [14]. As will be shown below, the fast and efficient hole transfer to the deep QW takes place even at low excess energies demonstrating that the photo-Dember effect is not a decisive factor for the low-temperature hole transfer to the deep QW.

The parameter that defines ballistic transport is the meanfree path $l_{b}$, which, in the classical picture, is related to the scattering time $\tau_{\mathrm{sc}}$ via $l_{b}=\bar{v} \tau_{\mathrm{sc}}$ (here $\bar{v}$ is the average thermal velocity). At low temperatures, the main carrier scattering mechanism is by acoustic phonons [15]. Moreover, it is the only low-temperature scattering mechanism with a rate that experiences temperature dependence (linear for both deformation potential and piezoelectric scattering [16]). If we assume that the number of holes reaching the deep QW is proportional to the mean-free path and the scattering time, the temperature dependence shown in Fig. 2 agrees with the one expected for the acoustic phonon scattering.

As the temperature is raised over $\sim 80 \mathrm{~K}$, the hole ballistic transport over the shallow QWs ceases. This is a consequence of the increased number of longitudinal optical (LO) phonons. For electrons, LO phonon scattering becomes the dominating scattering mechanism at $\sim 150 \mathrm{~K}$ [17]. Since the scattering time for holes is smaller (it depends on the effective mass $m^{*}$ as $m^{*-1 / 2}$ ), the hole-LO phonon scattering mechanism should become dominant at lower temperatures. When hole scattering with LO phonons becomes the most effective scattering mechanism, it starts limiting the mean-free path. When this parameter decreases to about a QW width, holes cannot traverse transport QWs without being captured, and their ballistic transport stops.

The ratio $n_{d} / n_{\mathrm{sh}}$ at $4 \mathrm{~K}$ is similar for five shallow QW structures with narrow $(5.5 \mathrm{~nm})$ and medium $(8.5 \mathrm{~nm})$ barriers (Fig. 2), and the three shallow QW medium barrier structures. For the wide $(12.5 \mathrm{~nm})$ barrier structure, it is about 2 times lower. This dependence on the barrier width allows estimating the low-temperature mean-free path $l_{b}$ for the hole transport. First, it is obvious that $l_{b}$ must be larger than the well width, $3 \mathrm{~nm}$, because otherwise the holes would be captured and stay in the first shallow QW. On the other extreme, $l_{b}$ could be compared to the whole shallow MQW region, $35-78 \mathrm{~nm}$ for the different structures. However, if the mean-free path were similar to the width of the MQW region, one would 
expect different $n_{d} / n_{\mathrm{sh}}$ values for the three and five shallow QW structures with medium barriers because of the different widths of the MQW region. This is not experimentally observed: the ratio $n_{d} / n_{\mathrm{sh}}$ in these structures is the same. The third characteristic length is that of a single shallow QW and adjacent barrier $(8.5 \mathrm{~nm}$ for the thin, $11.5 \mathrm{~nm}$ for medium, and $15.5 \mathrm{~nm}$ for thick barrier structures). Comparison of the relative number of carriers transferred to the deep QW in different structures suggests that $l_{b}$ is similar to the well and barrier width for the thin and medium barrier structures, i.e., $\sim 10 \mathrm{~nm}$. Such a value is also consistent with the lower $n_{d} / n_{\mathrm{sh}}$ value for the thick barrier structure.

It would be interesting to compare our result with the mean-free path evaluated from mobility $(\mu)$ measurements since $l_{b}=\bar{v} \mu \mathrm{m}^{*} / q$ (here $q$ is the elementary charge). Regretfully, there are no experimental data on the hole mobility in undoped or lightly $n$-type $\mathrm{GaN}$ or $\mathrm{InGaN}$ at low temperatures. At $\sim 250 \mathrm{~K}$, the highest reported hole mobility values are $400-500 \mathrm{~cm}^{2} /(\mathrm{Vs})[18,19]$. Another unknown parameter in the equation for the low-temperature mean-free path is the average hole thermal velocity (see discussion below). Assuming that the low-temperature hole mobility is the same as at $250 \mathrm{~K}\left(500 \mathrm{~cm}^{2} /(\mathrm{Vs})\right)$ and using the thermal velocity of $4-6 \times 10^{6} \mathrm{~cm} / \mathrm{s}$ corresponding to hole temperatures of 50 $100 \mathrm{~K}$, the mean-free path is $18-26 \mathrm{~nm}$, which is similar to our estimated value.

Recent Monte Carlo simulations of carrier dynamics in GaN [20] have produced heavy- and light-hole mean-free paths that are an order of magnitude smaller than our evaluation. These calculations, however, were performed for room temperature at which holes are effectively scattered by LO phonons. This result is consistent with our findings that at high temperatures the hole transport takes place via thermionic diffusion and not in a ballistic manner.

If the mean-free path is close to the well and barrier width, one could raise a question how would holes overcome triangular potential barriers at the shallow well/barrier interfaces (inset to Fig. 2). To explain this effect, one should recall that carriers are excited high into the bands with an electronhole pair excess energy of $1.27 \mathrm{eV}$. Due to the very efficient carrier-LO phonon scattering with a typical scattering time of 10-20 fs [20], within 100 fs for holes and $200 \mathrm{fs}$ for electrons [21] the carrier excess energy is reduced to below the energy of an LO phonon. Subsequent carrier cooling takes place with a much slower acoustic phonon emission on a 100-ps timescale [22]. Thus, photoexcited holes that participate in the ballistic transport have a considerable kinetic energy that enables overcoming the potential barriers at the well/barrier interfaces. This also means that at low temperatures the carrier temperature is higher than that of the lattice.

The elevated hole temperature should not only aid overcoming the interface potential barriers but should also affect the mean-free path via population of the higher hole bands since the rate of acoustic phonon scattering is proportional to the effective mass [15]. Because the effective masses for the InGaN QW continuum states are not known, we will only consider the barrier material $(\mathrm{GaN})$. At higher hole temperatures not only the heavy-hole band with the effective mass of $1.76 m_{0}$ [23] but also the light-hole band $\left(0.42 m_{0}\right.$, energy splitting from the heavy-hole band $6 \mathrm{meV}$ ) and, possibly, the

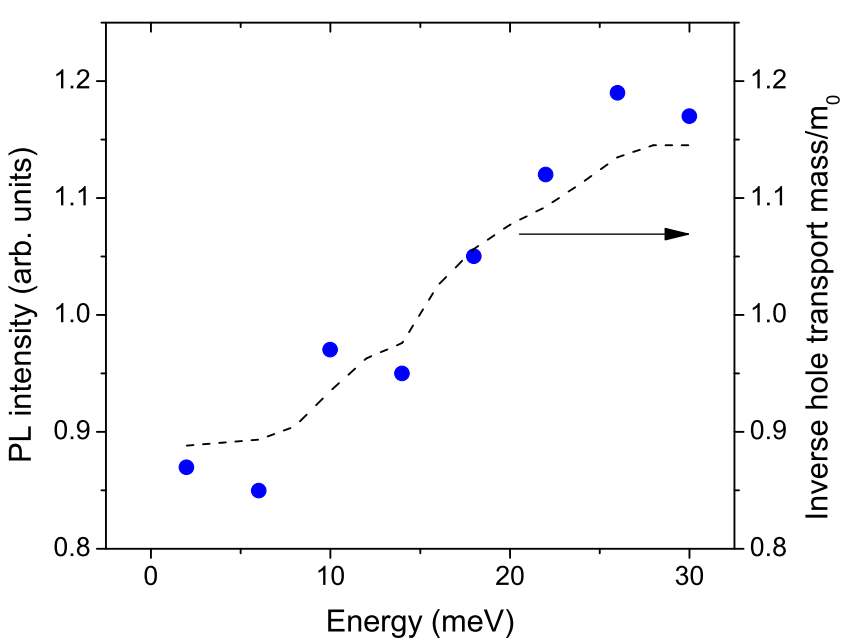

FIG. 4. Deep QW PL intensity at $4 \mathrm{~K}$ measured at different hole excess energies. The dashed curve shows an estimation of the inverse effective hole transport mass.

split-off band $\left(0.30 m_{0}, 21 \mathrm{meV}\right)[23,24]$ are populated. For carrier excitation with high excess energies, population of the different valence bands cannot be evaluated in a simple way because of the complex time-dependent carrier-carrier and carrier-phonon scattering. To test the influence of the hole excess energy, experiments with excitation energies just above the $\mathrm{GaN}$ band gap (3.503 eV at $4 \mathrm{~K}$ [25]) were performed.

The measurements were carried out at $4 \mathrm{~K}$ and low photoexcited carrier density. The hole excess energy was estimated from the excitation wavelength, GaN band gap, and heavy hole and electron effective masses $\left(0.19 m_{0}\right.$ in the latter case [26]). Exciting pulses had a linewidth of $\sim 20 \mathrm{meV}$; thus, excitation from the heavy- and light-hole bands could not be separated. However, while at the lowest photon energies excitation takes place only from the two topmost valence bands, at higher energies the split-off band is also involved. Since this band has a small effective mass, one would expect that at higher energies the rate for hole-acoustic phonon scattering would be smaller and the mean-free path longer.

Dependence of the deep QW PL intensity on the hole excess energy for the thin barrier structure is shown in Fig. 4. The excitation energies were above excitonic resonances in the featureless region of the absorption spectrum; hence, the PL energy dependence should reflect properties of the carrier transfer to the deep QW. The figure also shows an estimation of the inverse effective transport mass, which increases with excess energy due to the population of the light hole and splitoff bands. The transport mass (the average mass of the three valence bands weighted by corresponding population factors) was estimated assuming that photoexcited electrons, after emission of the possible amount of LO phonons, thermalize with holes, and that the population of the valence bands follows the Boltzmann distribution. The result was smoothed in order to account for the laser pulse width leveling out carrier temperature variations induced by $\mathrm{LO}$ phonon emission by electrons.

Figure 4 shows that spectrum of the deep QW PL intensity follows the trend of the inverse transport mass supporting the assumption that the ballistic hole transport is aided by the 
occupation of the light hole and split-off bands. A thorough modeling of the experimental results would require complex Monte Carlo calculations that take into account spatially, temporarily, and spectrally nonuniform carrier generation, carriercarrier, and carrier-phonon scattering effects and dynamical band profile variation, which is beyond the scope of this paper.

In conclusion, a large enhancement of the PL intensity from a deep QW in $n$-type $\mathrm{InGaN} / \mathrm{GaN}$ MQW structures designed to measure interwell carrier transport was observed at low temperatures. The temperature dependence of the relative number of holes in different wells and the fast hole transfer times suggest that hole transfer over the MQW structure takes place via ballistic transport with a mean-free path of about $10 \mathrm{~nm}$.

The authors are grateful to Prof. M. Shur for valuable comments. Research at KTH was financially supported by the Swedish Energy Agency (Contract No. 45390-1). Work at UCSB was supported by the Solid State Lighting and Energy Electronics Center (SSLEEC), the DOE SSL Program through Award No. DE-EE0008204 (J. Chaddock Program Manager), and the Simons Foundation through Award No. 601952.
[1] A. David, M. J. Grundmann, J. F. Kaeding, N. F. Gardner, T. G. Mihopoulos, and M. R. Krames, Appl. Phys. Lett. 92, 053502 (2008).

[2] S. Marcinkevičius, R. Yapparov, L. Y. Kuritzky, Y.-R. Wu, S. Nakamura, S. P. DenBaars, and J. S. Speck, Appl. Phys. Lett. 114, 151103 (2019).

[3] D. S. Sizov, R. Bhat, A. Zakharian, K. Song, D. E. Allen, S. Coleman, and C. Zah, IEEE Sel. Top. Quantum Electron. 17, 1390 (2011).

[4] X. Ni, X. Li, J. Lee, S. Liu, V. Avrutin, A. Matulionis, Ü. Özgür, and H. Morkoç, Superlattices Microstruct. 48, 133 (2010).

[5] Y. D. Jho, J. S. Yahng, E. Oh, and D. S. Kim, Phys. Rev. B 66, 035334 (2002).

[6] S. Marcinkevičius, K. M. Kelchner, L. Y. Kuritzky, S. Nakamura, S. P. DenBaars, and J. S. Speck, Appl. Phys. Lett. 103, 111107 (2013).

[7] T. Langer, M. Klisch, F. A. Ketzer, H. Jönen, H. Bremers, U. Rossow, T. Meisch, F. Scholz, and A. Hangleiter, Phys. Stat. Solidi B 253, 133 (2016).

[8] R. Ivanov, S. Marcinkevičius, Y. Zhao, D. L. Becerra, S. Nakamura, S. P. DenBaars, and J. S. Speck, Appl. Phys. Lett. 107, 211109 (2015).

[9] S. Pau, J. Kuhl, M. A. Khan, and C. J. Sun, Phys. Rev. B 58, 12916 (1998).

[10] A. Vinattieri, F. Bogani, L. Cavigli, D. Manzi, M. Gurioli, E. Feltin, J.-F. Carlin, D. Martin, R. Butté, and N. Grandjean, Phys. Rev. B 87, 075202 (2013).

[11] A. T. Roberts, A. Mohanta, H. O. Everitt, J. H. Leach, D. Van Den Broeck, A. M. Hosalli, T. Paskova, and S. M. Bedair, Appl. Phys. Lett. 103, 181106 (2013).

[12] M. Binder, A. Nirschl, R. Zeisel, T. Hager, H.-J. Lugauer, M. Sabathil, D. Bougeard, J. Wagner, and B. Galler, Appl. Phys. Lett. 103, 071108 (2013).
[13] M. Shahmohammadi, W. Liu, G. Rossbach, L. Lahourcade, A. Dussaigne, C. Bougerol, R. Butté, N. Grandjean, B. Deveaud, and G. Jacopin, Phys. Rev. B 95, 125314 (2017).

[14] A. Reklaitis, J. Appl. Phys. 109, 083108 (2011).

[15] W. Knap, E. Borovitskaya, M. S. Shur, L. Hsu, W. Walukiewicz, E. Frayssinet, P. Lorenzini, N. Grandjean, C. Skierbiszewski, P. Prystawko, M. Leszczynski, and I. Grzegory, Appl. Phys. Lett. 80, 1228 (2002).

[16] I. Hsu and W. Walukiewich, J. Appl. Phys. 89, 1783 (2001).

[17] E. Tiras, O. Celik, S. Mutlu, S. Ardali, S. B. Lisesivdin, and E. Ozbay, Superlattices Microstruct. 51, 733 (2012).

[18] M. Rubin, N. Newman, J. S. Chan, T. C. Fu, and J. T. Ross, Appl. Phys. Lett. 64, 64 (1994).

[19] T. T. Mnatsakanov, M. E. Levinshtein, L. I. Pomortseva, S. N. Yurkov, G. S. Simin, and M. A. Khan, Solid State Electron. 47, 111 (2003).

[20] V. A. Jhalani, J.-J. Zhou, and M. Bernardi, Nano Lett. 17, 5012 (2017).

[21] D. J. Suntrup III, G. Gupta, H. Li, S. Keller, and U. K. Mishra, Appl. Phys. Lett. 105, 263506 (2014).

[22] D. Hägele, R. Zimmermann, M. Oestreich, M. R. Hofmann, W. W. Rühle, B. K. Meyer, H. Amano, and I. Akasaki, Phys. Rev. B 59, R7797 (1999).

[23] S. Shokhovets, G. Gobsch, and O. Ambacher, Appl. Phys. Lett. 86, 161908 (2005).

[24] K. Kornitzer, T. Ebner, K. Thonke, R. Sauer, C. Kirchner, V. Schwegler, M. Kamp, M. Leszczynski, I. Grzegory, and S. Porowski, Phys. Rev. B 60, 1471 (1999).

[25] B. Monemar, P. P. Paskov, T. Paskova, J. P. Bergman, G. Pozina, W. M. Chen, P. N. Hai, I. A. Buyanova, H. Amano, and I. Akasaki, Mater. Sci. Eng. B 93, 112 (2002).

[26] P. Rinke, M. Winkelnkemper, A. Qteish, D. Bimberg, J. Neugebauer, and M. Scheffler, Phys. Rev. B 77, 075202 (2008). 